\title{
Severe Hyponatremia From Water Intoxication Associated With Preparation for a Urine Flow Study
}

\author{
Michael Su ${ }^{\mathrm{a}}$, Henry H. Woo ${ }^{\mathrm{a}, \mathrm{b}, \mathrm{c}}$
}

\begin{abstract}
To discuss the natural history and impact of hyponatremia secondary to water intoxication in the setting of a urine flow study. Case report and literature review. An 82 year old male developed severe hyponatraemia following preparation for a urine flow study as part of ongoing follow up of lower urinary tract symptoms. He was admitted to an intensive care unit after developing acute confusion with a sodium of $114 \mathrm{mmol} / \mathrm{L}$ and discharged after his symptoms improved with slow correction of his sodium level. There are few reports in the literature of this event and no consensus guidelines on safe preparation for flow studies or other imaging modalities requiring pre-procedural fluid hydration. Patients with medications or conditions predisposing to hyponatremia are at higher risk of adverse events when overhydration occurs. Hyponatremia is a major adverse outcome of urine flow studies. Wider reporting of this condition is required to improve awareness of its effects and develop consensus guidelines on preventing its occurrence.
\end{abstract}

Keywords: Hyponatremia; Water intoxication; Urine flow study; Uroflowmetry; Flow study; Benign prostatic hypertrophy

\section{Introduction}

Water intoxication is a serious condition resulting from over-

Manuscript accepted for publication January 13, 2012

${ }^{a}$ Department of Urology, Westmead Hospital, Sydney, Australia

${ }^{\mathrm{b}}$ Sydney Adventist Hospital Clinical School, University of Sydney, Sydney, Australia

${ }^{c}$ Corresponding author: Henry H. Woo, P.O. Box 5017, Wahroonga,

NSW, 2076, Australia. Email: hwoo@urologist.net.au

doi:10.4021/jmc498w consumption of water leading to hyponatraemia. Patients can present with a range of symptoms from nausea, vomiting to confusion, seizures and loss of consciousness. The condition has been widely reported in the literature amongst endurance athletes $[1,2]$, psychiatric patients [3-11] and military recruits $[12,13]$. Water intoxication has also been reported in patients preparing for imaging studies that require fluid preparation such as abdominal or pelvic ultrasonography [14-18].

Urine flow studies are performed for patients with lower urinary tract symptoms and a history of prostate disease as an objective assessment of urinary function. Patients are generally instructed to drink one to two litres of fluid immediately prior to the procedure to fill the bladder in order to perform the study. However, there is limited data from the literature on overhydration leading to symptomatic hyponatraemia with only one report published on water intoxication in the setting of uroflowmetry [19]. We present a patient from our practice that developed severe symptoms from water intoxication as a result of excessive fluid intake prior to a urine flow study to improve awareness of this potential adverse outcome and stimulate discussion around developing practice guidelines on preventing this condition.

\section{Case Report}

An 82 year old Caucasian male with a previous history of TURP, AF, hypertension and depression was reviewed for ongoing follow up of chronic lower urinary tract symptoms. His symptoms had worsened since his last visit with his WHO International Prostate Symptom Score rising from 17 to 23 in six months. A flow study was performed and this demonstrated a peak urinary flow of $11.2 \mathrm{ml} / \mathrm{sec}$ on a voided volume of $142 \mathrm{ml}$ and the ultrasound measured post void residual volume was $100 \mathrm{ml}$. While preparing for the study the patient consumed approximately three litres of water over four hours.

Several hours after the study his family noticed that the patient had difficulty finding words and was progressively more confused. He was brought to a local emergency department and found to be acutely confused with a GCS of 14 . The physical examination was unremarkable aside from an 
ejection-systolic murmur with prosthetic heart sounds. His serum sodium level on admission was $114 \mathrm{mmol} / \mathrm{L}$ (normal range 135 - $145 \mathrm{mmol} / \mathrm{L})$. There were no acute infarcts or haemorrhage on a non-contrast CT brain scan. The patient was admitted to ICU for management of severe hyponatremia and commenced on an $800 \mathrm{ml}$ daily fluid restriction. Mirtazapine and ramipril were withheld during the admission. After several days of slow correction, his alertness and confusion improved. Seven days later he was discharged with a sodium level of $127 \mathrm{mmol} / \mathrm{L}$ and a diagnosis of hyponatraemia secondary to excessive fluid hydration exacerbated by tricyclic antidepressant therapy.

\section{Discussion}

The case presented demonstrates the importance of appropriate counselling when preparing patients for urine flow studies. Patients with predisposing medications or conditions should be warned of the risks of excessive fluid consumption before they begin to prepare for a study. Medications that are commonly known to cause hyponatraemia include diuretics (e.g. thiazides, indapamide, loop diuretics), anti-depressants (SSRIs, TCAs, monoamine oxidase inhibitors), anti-psychotics (e.g. haloperidol), anti-convulsants (e.g. sodium valproate, carbemazepine) and anti-neoplastic agents [20].

In rare instances, some commonly prescribed medications including ACE-inhibitors, amlodipine, proton-pump inhibitors, amiodarone, trimethoprim-sulfamethoxazole and ciprofloxacin have also been known to cause SIADH-related hyponatraemia [20]. Patients with a history of congestive heart failure, malignancy, hepatic cirrhosis or nephrotic syndrome are at risk of developing hypervolaemic hyponatraemia with a large fluid load [21].

Our patient was on a regular tricyclic antidepressant (mirtazapine) and ACE inhibitor (ramipril) that were both identified as significant contributors to his hyponatraemia. These medications were withheld during his admission until further review by his family practitioner. There was a trend towards mild hyponatraemia on review of his previous biochemistry with a level of $132 \mathrm{mmol} / \mathrm{L}$ one month prior to his admission. This demonstrates the complexities of performing urine flow studies in a population with predisposing risk factors. Risk stratification within best practice guidelines will be necessary to identify patients undergoing such studies that would benefit most from tailored fluid intake volumes.

Hyponatraemia from overconsumption of fluid is an underreported adverse outcome of preparation for urinary flow studies with a risk of high morbidity and mortality. There is currently limited guidance on the quantity of water intake recommended for bladder preparation in both urine flow studies and abdominal or pelvic ultrasonography. Previous studies have recommended the need for standardised practice guidelines especially for patients at risk of serum inap- propriate $\mathrm{ADH}$ syndrome related hyponatraemia [18]. The case we have presented provides further evidence of the need for an improvement in current best practice. We recommend that a consensus guideline should be developed for patients undergoing urine flow studies on pre-study fluid consumption to minimise the incidence of future cases as demonstrated in this report.

\section{Grant Support}

None.

\section{References}

1. Noakes T. Hyponatremia in distance runners: fluid and sodium balance during exercise. Curr Sports Med Rep. 2002;1(4):197-207.

2. Noakes TD, Sharwood K, Collins M, Perkins DR. The dipsomania of great distance: water intoxication in an Ironman triathlete. Br J Sports Med. 2004;38(4):E16.

3. de Leon J, Verghese C, Tracy JI, Josiassen RC, Simpson GM. Polydipsia and water intoxication in psychiatric patients: a review of the epidemiological literature. Biol Psychiatry. 1994;35(6):408-419.

4. Korzets A, Ori Y, Floro S, Ish-Tov E, Chagnac A, Weinstein T, Zevin D, et al. Case report: severe hyponatremia after water intoxication: a potential cause of rhabdomyolysis. Am J Med Sci. 1996;312(2):92-94.

5. Sato D, Miyashita Y, Himura I, Tokuda N, Kase T, Tajima M, Sawamura Y, et al. [A case of water intoxication presenting as urinary retention in a psychotic patient]. Hinyokika Kiyo. 1997;43(12):883-886.

6. Yanagi N, Maruyama T, Uehata S, Wakimoto Y, Sasaki Y, Arita M. Electrical and mechanical abnormalities in the heart of a schizophrenic patient with hyponatremia derived from water intoxication. J Cardiol. 1998;32(3):197-204.

7. Umansky L, Sella A. [Psychogenic polydipsia leading to water intoxication]. Harefuah. 2000;138(1):9-12, 87.

8. Gonzalez I, Perez N, Penas LEM, A LL, de la Rubia A. High risk of polydipsia and water intoxication in schizophrenia patients. Schizophr Res. 2008;99(1-3):377-378.

9. Vandepitte M, Vandereycken W. [Water intoxication in two girls with anorexia nervosa]. Tijdschr Psychiatr. 2008;50(8):545-548.

10. Goldman MB. The mechanism of life-threatening water imbalance in schizophrenia and its relationship to the underlying psychiatric illness. Brain Res Rev. 2009;61(2):210-220.

11. Funayama M, Hisamatsu T, Koreki A. Central pontine demyelinolysis following water intoxication in schizophrenia. Schizophr Res. 2011;125(2-3):300-301. 
12. Gutmann FD, Gardner JW. Fatal water intoxication of an Army trainee during urine drug testing. Mil Med. 2002;167(5):435-437.

13. Tilley MA, Cotant CL. Acute water intoxication during military urine drug screening. Mil Med. 2011;176(4):451453.

14. Christenson LL, Scott D. Acute water intoxication following pelvic ultrasound examination. Postgrad Med. 1985;77(3):161-162.

15. Shapira I, Isakov A, Almog C. Hyponatremia as the result of preparation for abdominal ultrasound examination. J Clin Ultrasound. 1988;16(1):61-62.

16. Bhargava R, Lewandowski BJ. Water intoxication: a complication of pelvic US in a patient with syndrome of inappropriate antidiuretic hormone secretion. Radiology. 1991;180(3):723-724.

17. Camkurt MA, Coskun F, Aksu NM, Akpinar E, Ay D.
Iatrogenic water intoxication after pelvic ultrasonography imaging. Am J Emerg Med. 2010;28(3):385 e381383.

18. Yalcin-Cakmakli G, Karli Oguz K, Shorbagi A, Funda Bas D, Ergan-Arsava B, Kunt M, Akif Topcuoglu M. Hyponatremic encephalopathy after excessive water ingestion prior to pelvic ultrasound: neuroimaging findings. Intern Med. 2010;49(16):1807-1811.

19. Issa MM, Pruthi RS, Vial C, McNamara DE, Terris MK. An unusual complication following uroflowmetry: water intoxication resulting in hyponatremia and seizure. Urol Int. 1997;59(2):129-130.

20. Liamis G, Milionis H, Elisaf M. A review of drug-induced hyponatremia. Am J Kidney Dis. 2008;52(1):144153.

21. Lien YH, Shapiro JI. Hyponatremia: clinical diagnosis and management. Am J Med. 2007;120(8):653-658. 